ARTICLE

\title{
A substrate-bound structure of cyanobacterial biliverdin reductase identifies stacked substrates as critical for activity
}

Haruna Takao ${ }^{1,2}$, Kei Hirabayashi ${ }^{1}$, Yuki Nishigaya ${ }^{3}$, Haruna Kouriki ${ }^{1}$, Tetsuko Nakaniwa ${ }^{4}$, Yoshinori Hagiwara ${ }^{5}$, Jiro Harada ${ }^{6}$, Hideaki Sato ${ }^{6}$, Toshimasa Yamazaki ${ }^{3}$, Yoichi Sakakibara7, Masahito Suiko7, Yujiro Asada ${ }^{8}$, Yasuhiro Takahashi ${ }^{9}$, Ken Yamamoto ${ }^{6}$, Keiichi Fukuyama4,10, Masakazu Sugishima ${ }^{6}$ \& Kei Wada ${ }^{1}$

Biliverdin reductase catalyses the last step in haem degradation and produces the major lipophilic antioxidant bilirubin via reduction of biliverdin, using $N A D(P) H$ as a cofactor. Despite the importance of biliverdin reductase in maintaining the redox balance, the molecular details of the reaction it catalyses remain unknown. Here we present the crystal structure of biliverdin reductase in complex with biliverdin and NADP ${ }^{+}$. Unexpectedly, two biliverdin molecules, which we designated the proximal and distal biliverdins, bind with stacked geometry in the active site. The nicotinamide ring of the NADP ${ }^{+}$is located close to the reaction site on the proximal biliverdin, supporting that the hydride directly attacks this position of the proximal biliverdin. The results of mutagenesis studies suggest that a conserved Arg185 is essential for the catalysis. The distal biliverdin probably acts as a conduit to deliver the proton from Arg185 to the proximal biliverdin, thus yielding bilirubin.

\footnotetext{
${ }^{1}$ Organization for Promotion of Tenure Track, University of Miyazaki, Miyazaki 889-1692, Japan. ${ }^{2}$ Graduate School of Medicine and Veterinary Medicine, University of Miyazaki, Miyazaki 889-1692, Japan. ${ }^{3}$ Advanced Analysis Center, National Agriculture and Food Research Organization, Ibaraki 305-8602, Japan. ${ }^{4}$ Department of Biological Sciences, Graduate School of Science, Osaka University, Osaka 560-0043, Japan. ${ }^{5}$ Department of Biochemistry and Applied Chemistry, National Institute of Technology, Kurume College, Fukuoka 830-8555, Japan. ${ }^{6}$ Department of Medical Biochemistry, Kurume University School of Medicine, Fukuoka 830-0011, Japan. ${ }^{7}$ Department of Biochemistry and Applied Biosciences, Faculty of Agriculture, University of Miyazaki, Miyazaki 889-2192, Japan. ${ }^{8}$ Department of Pathology, Faculty of Medicine, University of Miyazaki, Miyazaki 889-1692, Japan. ${ }^{9}$ Division of Life Science, Graduate School of Science and Engineering, Saitama University, Saitama 338-8570, Japan. ${ }^{10}$ Department of Applied Chemistry, Graduate School of Engineering, Osaka University, Osaka 565-0871, Japan. Correspondence and requests for materials should be addressed to M.S. (email: sugishima_masakazu@med.kurume-u.ac.jp) or to K.W. (email: keiwada@med.miyazaki-u.ac.jp).
} 
B iliverdin reductase (BVR, EC 1.3.1.24), first discovered in the 1960s (ref. 1), is the enzyme that converts biliverdin IX $\alpha$ $(\mathrm{BV})$, a product of haem degradation, to bilirubin IX $\alpha(\mathrm{BR})$; this product is a bile pigment and a source of jaundice. Because BR forms intra-molecular hydrogen bonds, this reduction reaction causes the bilin pigment to change from hydrophilic to lipophilic ${ }^{2}$. Neonatal jaundice is a common disease in newborns; light therapy dramatically reduces the symptoms by changing the conformation of BR and breaking these intra-molecular hydrogen bonds, causing $\mathrm{BR}$ to become more hydrophilic and thus promoting its excretion ${ }^{3}$. Although over-accumulation of BR is toxic, at physiological concentrations $\mathrm{BR}$ is the major antioxidant responsible for protecting cells from $\mathrm{H}_{2} \mathrm{O}_{2}$ (ref. 4). Concomitant with $\mathrm{H}_{2} \mathrm{O}_{2}$-scavenging, $\mathrm{BV}$ produced by oxidation of $\mathrm{BR}$ is rapidly reduced back to $\mathrm{BR}$ by $\mathrm{BVR}$, thereby amplifying its antioxidant efficiency 10,000-fold even though the BR concentration in tissues is quite low $(\sim 20-50 \mathrm{nM}:<0.1 \%$ level as compared with glutathione $)^{5}$. The depletion of BVR by RNA interference markedly increases the level of reactive oxygen species and causes apoptotic cell death ${ }^{4}$. Thus, BVR plays a crucial role in the maintenance of intracellular redox balance.

BVR catalyses the reduction of the $\mathrm{C} 10$ double bond $(\gamma$-methene bridge) of $\mathrm{BV}$ using $\mathrm{NAD}(\mathrm{P}) \mathrm{H}$ to produce $\mathrm{BR}$, a reaction in which the hydride $\left(\mathrm{H}^{-}\right.$: a proton and two electrons) is donated by $\mathrm{NAD}(\mathrm{P}) \mathrm{H}$ (Fig. 1). This reaction also requires one additional proton to reduce the $\mathrm{C} 10$ double bond, but this proton donor has remained enigmatic for the past 50 years. In other words, the catalytic residue in the BVR protein moiety remains to be identified. Several crystallographic analyses have been performed to date: the structures of rat BVR in the apo-form and in complex with $\mathrm{NAD}^{+}$have been reported by two independent groups ${ }^{6,7}$, and the structure of human BVR in complex with NADP ${ }^{+}$has been deposited in the RCSB Protein Data Bank (PDB ID: 2H63). These crystallographic analyses revealed the binding site and mode of $\mathrm{NAD}(\mathrm{P})^{+}$. Although the substrate-binding site remained unclear, the residues located around the nicotinamide ring of $\mathrm{NAD}^{+}$were considered strong candidates for the catalytic residues. Unexpectedly, however, extensive site-directed mutagenesis experiment revealed that alteration of these residues did not affect enzymatic activity. One exception was Tyr97 in rat BVR, which is located in the immediate vicinity of the nicotinamide ring; mutation of this residue reduces activity by $50 \%$ (ref. 7 ). Thus, Tyr97 promotes the enzymatic reaction, probably by indirectly influencing hydride transfer from $\mathrm{NAD}(\mathrm{P}) \mathrm{H}$, but is not essential for catalysis.

Other characterizations of the mechanism underlying the BVR-catalysed reaction have been carried out, for example, investigations of substrate specificities using synthesized tetrapyrrole compounds ${ }^{8-10}$. These experiments examined a wide variety of tetrapyrrole compounds, including not only structurally modified alkyl side chains of tetrapyrrole but also BV isomers. The results revealed the invariant features of the BVR substrate: BVR exhibits a broad bilin substrate specificity, but strictly requires a propionate as the tetrapyrrole side chains ${ }^{11}$. Furthermore, a carboxyl group with a dissociable proton on the propionate side chain is essential, because its methyl- or azo-esterification completely abolishes BVR activity ${ }^{12}$.

Here we report the X-ray structures of the apo-, NADP ${ }^{+}$. bound and BV-NADP ${ }^{+}$complex forms of BVR. This is the structure of the substrate-cofactor-enzyme ternary complex of BVR, revealing a binding mode in which two biliverdin molecules bind with stacked geometry in the active site. This structure and the results of site-direct mutagenesis can explain how BVR reduces $\mathrm{BV}$ to $\mathrm{BR}$, thus resolving a longstanding mystery regarding the BVR reaction mechanism.

\section{Results}

Overall structure of apo-BVR from cyanobacteria. In this study, we used a cyanobacterial BVR from Synechocystis sp. PCC 6803 $(\text { Syn BVR })^{13}$ as a target for the structural analyses. The detailed properties of the purified Syn BVR, such as oligomeric state, are described in Supplementary Note 1 and Supplementary Fig. 1. All previous trials aimed at determining the structure of the substrate-binding site of rat BVR were unsuccessful ${ }^{6,7}$. Syn BVR is composed of 328 residues and shares more than $20 \%$ sequence identity with rat and human BVR proteins (Supplementary Fig. 2). We determined the crystal structure of Syn BVR by the single isomorphous replacement method coupled with anomalous scattering method using an Au-derivative crystal. The structure of apo-Syn BVR was refined at $2.1 \AA$ resolution to $R$ and $R_{\text {free }}$ values of 0.200 and 0.239 , respectively (Table 1 ). The asymmetric unit contains two BVR molecules. Although the electron density for apo-Syn BVR was mostly of high quality and continuous, the densities for the $\mathrm{N}$-terminal region (residues 1-8) and C-terminal region (residues 326-328) were poorly defined. Therefore, these residues were not included in the model. Syn BVR consists of two structural domains (N-terminal and C-terminal domains), with the active sites in an obvious cleft between them (see Fig. 2). The $\mathrm{N}$-terminal domain has a typical Rossmann fold, in which a parallel $\beta$-sheet (strands 1-5) is surrounded by five helices (helices 1-5). The C-terminal domain contains eight $\beta$-strands (strands 6-13) and six $\alpha$-helices (helices 6-11). The overall topology of Syn BVR is nearly the same as those of the rat and human homologues, with two minor differences: Syn BVR has an additional short $\alpha$-helix ( $\alpha 2)$ in the $\mathrm{N}$-terminal domain, and has three additional $\beta$-strands in the C-terminal domain (Supplementary Fig. 2).

NADP ${ }^{+}$binding mode. The $F_{\mathrm{o}}-F_{\mathrm{c}}$ electron density map calculated from the $\mathrm{NADP}^{+}$-soaked apo-form crystal clearly indicates that $\mathrm{NADP}^{+}$binds in the cleft between the two domains (Fig. 3a). The density is visible for only one molecule in the asymmetric unit; thus, the two crystallographically independent BVR proteins in the asymmetric unit comprise one apo-form and one $\mathrm{NADP}^{+}$-bound form. The electron density of bound

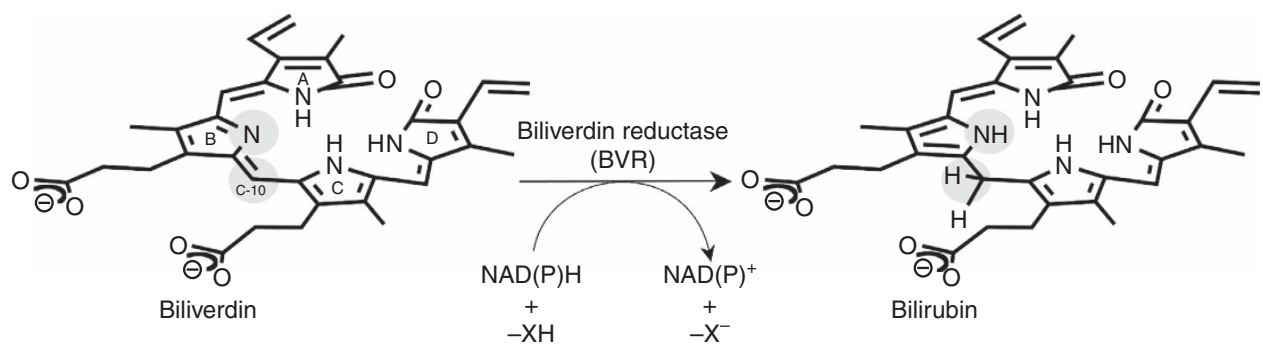

Figure 1 | Reaction catalysed by BVR. The C10 double bond ( $\gamma$-methene bridge) in BV is reduced by hydride from the cofactor, and the pyrrole nitrogen of the ring is protonated (hydride and proton are depicted on grey circular backgrounds on the product, BR). 


\section{Table 1 | Data collection and refinement statistics.}

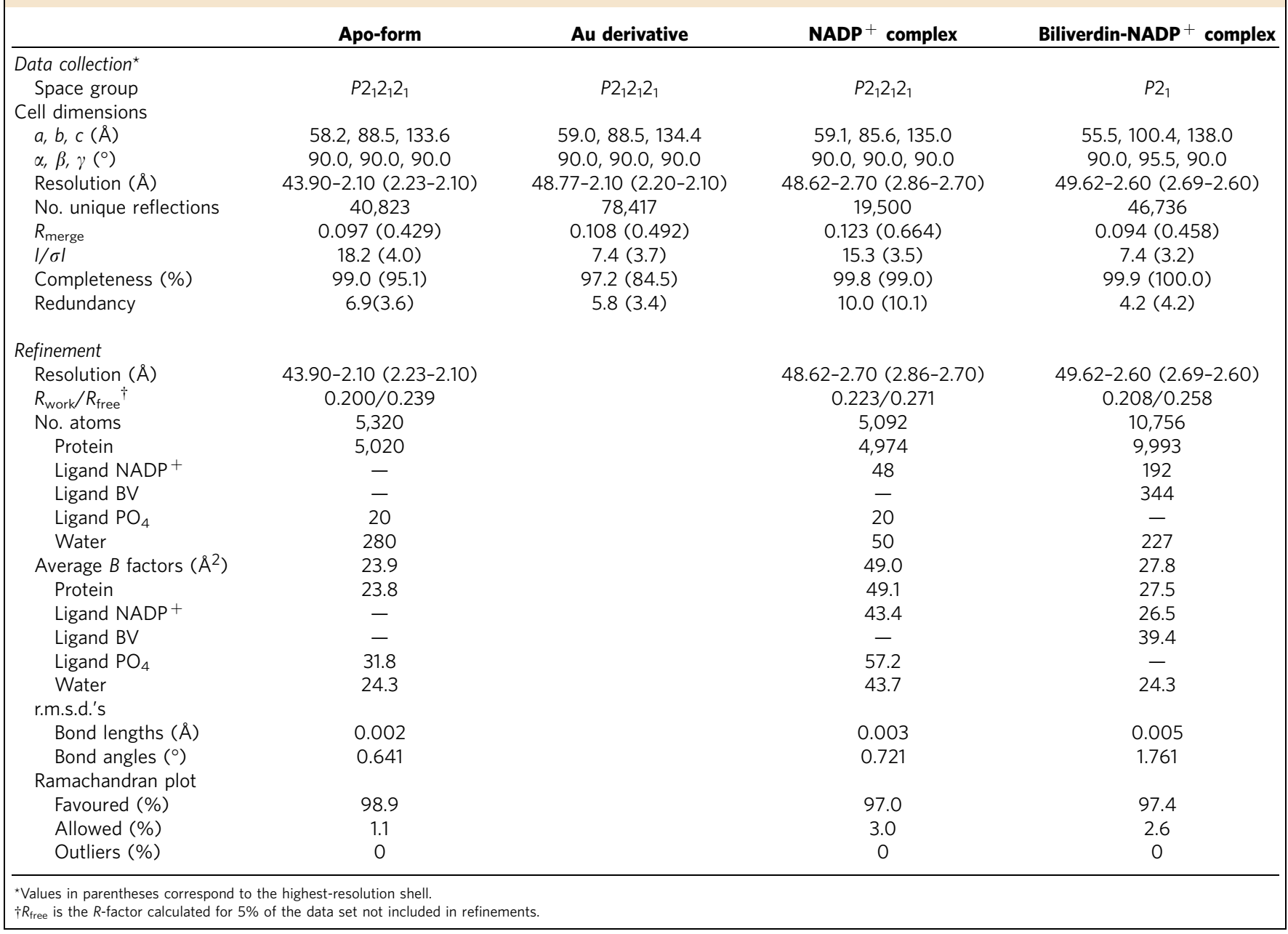

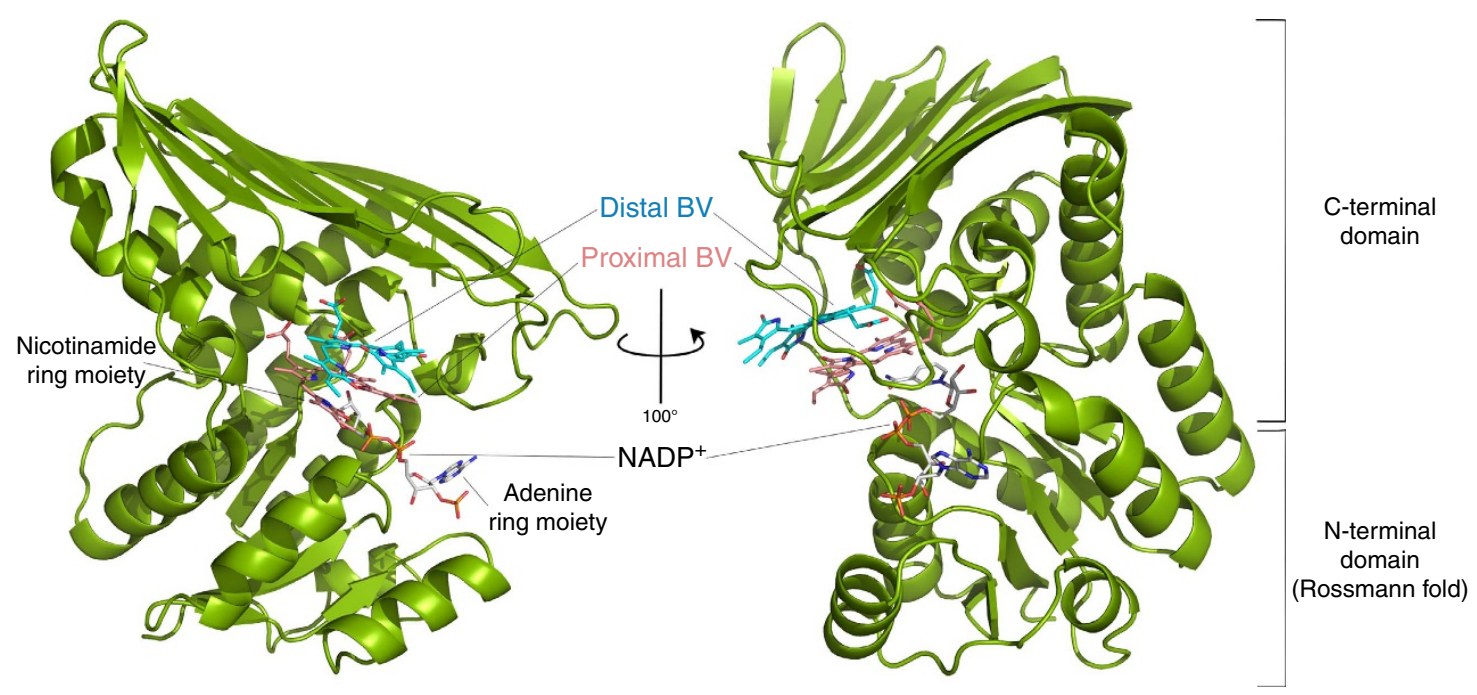

Figure 2 | Overall structure of the Syn BVR complex. Ribbon representation of the crystal structure of Syn BVR in complex with BV and NADP + . The bound BVs and NADP ${ }^{+}$are depicted with sticks. Right, View rotated by $100^{\circ}$ about the vertical axis relative to left panel.

$\mathrm{NADP}^{+}{ }^{+}$was sufficiently clear that conformation and orientation could be explicitly determined. $\mathrm{NADP}^{+}$is held by hydrogen bonds and hydrophobic interactions; Thr18, Ala21, Ser44, Arg167 and Trp168 form hydrogen bonding (Fig. 3a), whereas Trp41, Ile80, Glu101 and Tyr102 are involved in hydrophobic contact.
Comparisons between the apo-form and the $\mathrm{NADP}^{+}$-bound form revealed small but significant structural rearrangements upon $\mathrm{NADP}^{+}$binding: the N-terminal domain shifted $\sim 1.0 \AA$ in order to expand the active site (Supplementary Fig. 3), and the loop between $\beta 5$ and a part of helix $\alpha 6$ (residues 129-135) moved 

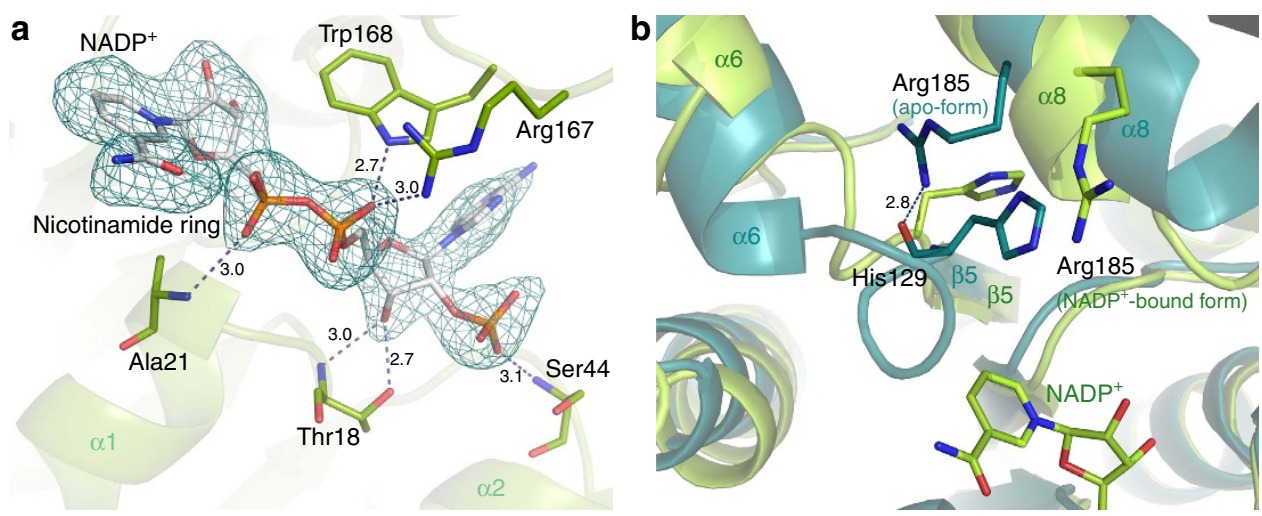

Figure 3 | Electron density map around the bound NADP ${ }^{+}$in the Syn BVR. (a) An omit $F_{\mathrm{o}}-F_{\mathrm{c}}$ difference map for NADP ${ }^{+}$contoured at $2.0 \sigma(c y a n)$ is overlaid on stick models of Syn BVR and the bound NADP ${ }^{+}$. Dashed lines indicate hydrogen bonds, and distances are represented in angstrom units. (b) Structural rearrangements in the active site upon NADP ${ }^{+}$binding. The structures of the apo-form (teal green) and NADP ${ }^{+}$-bound form (light green) are superposed.

$\sim 2.0 \AA$ away from the nicotinamide ring of the bound $\mathrm{NADP}^{+}$. Because of the steric hindrance configuration of the corresponding site in the apo-form would not able to bind NADP ${ }^{+}$; accordingly, these movements reflect an 'induced fit' type of structural change. This induced fit was probably triggered by yet other structural rearrangements. Specifically, the regions around helix $\alpha 8$ differed from those in the apo-form (Fig. 3b): the starting point of helix $\alpha 8$, located near helix $\alpha 6$ as well as the nicotinamide ring of the bound $\mathrm{NADP}^{+}$(but not directly interacting with $\mathrm{NADP}^{+}$), was altered. In the apo-form, the guanidine group of Arg185 in helix $\alpha 8$ formed a hydrogen bond with the carbonyl oxygen of His129 in the loop. In the $\mathrm{NADP}^{+}$-bound form, the hydrogen bond between Arg185 and His129 was broken, and the side chain of the Arg185 rotated toward the active site due to the overall rearrangement. These structural changes confer not only substrate-binding ability, but also catalytic activity, on BVR (see below).

Substrate BV binding mode. We successfully obtained BV-bound BVR crystals by the co-crystallization screening, and the resulting crystals were a bluish-green colour (Fig. 4a). The BV-bound crystals belong to a different space group $\left(P 2_{1}\right)$ than those of the apo- and $\mathrm{NADP}^{+}$soaked forms $\left(P 2_{1} 2_{1} 2_{1}\right)$, and the asymmetric unit contains four BVR molecules (A to D molecules). Surprisingly, two BV molecules are bound to the catalytic cleft between the two domains (Fig. 2), and adopt a stacked configuration along with the tetrapyrrole plane (Fig. 5). In each of the four BVR molecules, electron densities derived from the tetrapyrrole moiety of two BV molecules are clear (Fig. 5a). $\mathrm{NADP}^{+}$molecules were also bound by all four BVR molecules, although we did not add the $\mathrm{NADP}^{+}$reagent during the purification and crystallization procedures. Therefore, the $\mathrm{NADP}^{+}$in the BV-bound crystal was derived from E. coli, and their binding configurations are thought to represent the authentic form. The configuration of $\mathrm{NADP}^{+}$in the ternary complex was identical to that in the $\mathrm{NADP}^{+}$-soaking crystal. This structure is the substrate-cofactor-enzyme ternary complex reported for BVR. One BV (the proximal BV) was bound in close vicinity of the nicotinamide ring, and the other one (the distal BV) was located on the upper stack with the proximal BV (Fig. 5).

The superimposition of four BVR molecules in the asymmetric unit showed that the binding configurations of $\mathrm{NADP}^{+}$and proximal and distal BVs are almost the same. The proximal BV interacts with the distal BV by hydrophobic and van der Waals interactions. The propionate groups form two salt bridges with amino acid residues (Fig. 5b), one interacting with Arg185, and
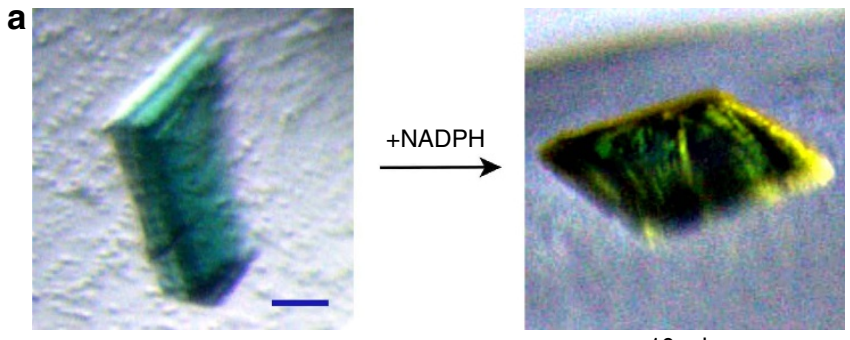

b
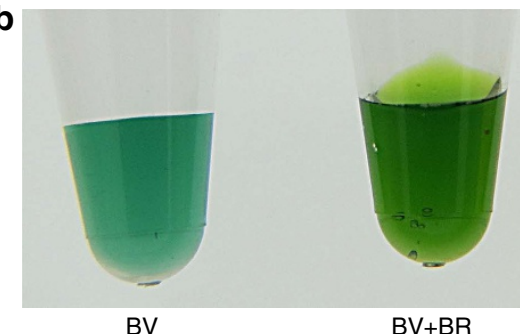

$10 \mathrm{~min}$

Figure 4 | The enzymatic reaction in the crystalline state. (a) The BV reduction could proceed in the crystal by adding NADPH. Right, 10 min after the reaction. The bluish-green colour in the crystal (left) is derived from the bound BV. The yellow-green colour in the crystal (right) is probably derived from the mixture of BV and BR. Scale bar, $20 \mu \mathrm{m}$ (b) The colours of the pigments in solution, provided as a reference: $B V$ (left); mixture of $B V$ and $\mathrm{BR}$ (middle) and BR (right). Final concentrations of all pigments were adjusted to $0.5 \mathrm{mM}$.

the other interacting with Arg188. These interactions between the propionate group and the surrounding residues fix the propionate side chains to these positions. The $\mathrm{C} 10$ double bond in the proximal $\mathrm{BV}$, which is to be reduced, faces the nicotinamide $\mathrm{C} 4$ carbon (origin of the hydride) of the $\mathrm{NADP}^{+}$; the distance between the $\mathrm{C} 10$ carbon of the proximal $\mathrm{BV}$ and the $\mathrm{C} 4$ carbon of the $\mathrm{NADP}^{+}$is $2.6 \AA$, and the angle between the $\mathrm{C} 10$ and nicotinamide ring was $\sim 120^{\circ}$. This configuration of the proximal BV appears suitable to accept the hydride from the nicotinamide ring, and strongly suggests that the hydride is directly transferred to the $\mathrm{C} 10$ carbon in the proximal BV.

In the distal $\mathrm{BV}$, the binding configuration could be also defined based on the electron density map (Fig. 5a). The configurations of all four distal BVs in the asymmetric unit are almost identical. The tetrapyrrole plane of the distal BV is located nearly parallel to that of the proximal $\mathrm{BV}$, and the directions of the propionate side chains of each BV are the same. The distal BV 

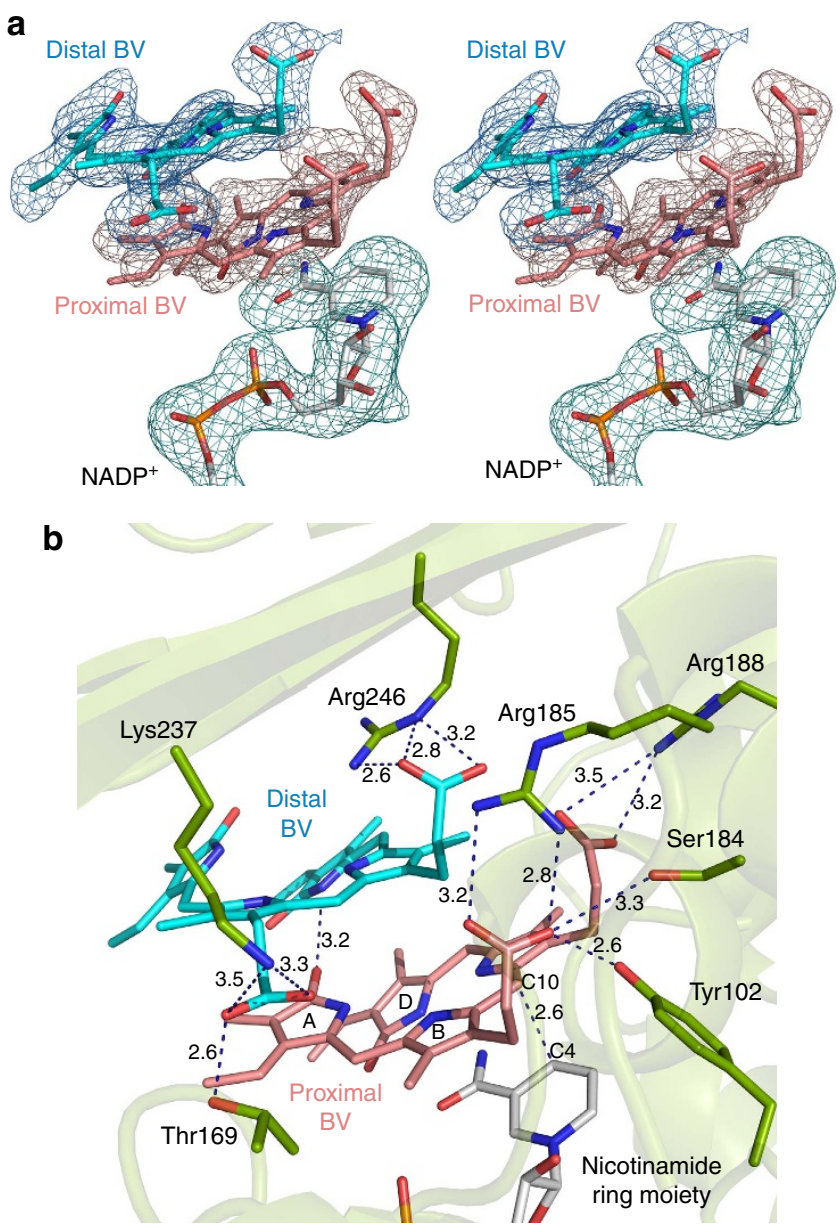

Figure 5 | The binding modes of the BVs and NADP ${ }^{+}$in Syn BVR.

(a) Stereo view of the electron density map around the bound two BVs and $\mathrm{NADP}^{+}$in the Syn BVR. An omit $F_{\mathrm{o}}-F_{\mathrm{c}}$ difference map for BVs (blue and salmon pink) and $\mathrm{NADP}^{+}$(cyan) contoured at $2.0 \sigma$ is overlaid on the stick models of the bound $\mathrm{BVs}_{\mathrm{s}}$ and the bound $\mathrm{NADP}^{+}$. The view direction is same as in right panel in Fig. 2. (b) The environment around the bound two BVs in the Syn BVR. The bound BVs, NADP ${ }^{+}$and the residues involved in the BV binding are shown with the stick. Dashed lines indicate hydrogen bonds.

is located $\sim 4 \AA$ above, translated by $\sim 2.5 \AA$ to the solvent side and rotated $\sim 45^{\circ}$ relative to the proximal BV (Fig. 5b). Consequently, the B-ring in the distal BV is located just above the A-ring of the proximal BV. One propionate side chain forms a salt-bridge with the Arg246, and the other side chain forms a hydrogen bond with Thr169. In addition, the pyrrole nitrogen of the D-ring in the distal BV might form a hydrogen bond with the lactam group $(\mathrm{C}=\mathrm{O})$ of the $\mathrm{A}$-ring in the proximal $\mathrm{BV}$; the distance between them was $\sim 3.2 \AA$.

Steady-state kinetics of Syn BVR. We next investigated the catalytic residue based on this structure. One proton and two electrons are served from the $\mathrm{NAD}(\mathrm{P}) \mathrm{H}$ directly to the $\mathrm{C} 10$ double bond in the proximal BV; thus, the other unidentified catalytic residue must be located close to the proximal BV. The structure, however, revealed that only the hydroxyl group of Tyr102 interacts with $\mathrm{C} 10$, and this interaction is weak $(\sim 3.5 \AA$, either via van der Waals force or $\mathrm{CH} / \mathrm{O}$ hydrogen bond). Previous mutagenesis studies of Syn and rat BVRs clearly indicated that the tyrosine residue (Tyr97 in rat BVR) is not a direct proton donor ${ }^{6,7,14}$. Although the H84A and D287A mutations in Syn
BVR dramatically reduced its activity ${ }^{14}$, these residues have no direct interaction with bound $\mathrm{NADP}^{+}$or bound $\mathrm{BV}$ (Supplementary Fig. 4). Accordingly, we excluded His84 and Asp287 as candidates for the proton donor (see details in Supplementary Note 2 and Supplementary Fig. 4). Therefore, we further investigated the possible candidates for the catalytic residues surrounding the proximal and distal BVs. Six residues were considered as possible proton donors (within $\sim 3.5 \AA$ ): the hydroxy groups of Thr169 and Ser184, and the amino groups of Arg185, Arg188, Lys237 and Arg246 (Fig. 5b).

Steady-state kinetics, monitored using the Syn BVR proteins containing mutations in these candidate proton donor residues, revealed the plausible catalytic residues (Table 2). The enzymatic activity of the mutation at Tyr 102 was reduced to $~ 40 \%$ in terms of $k_{\text {cat }}$ value. This behaviour was in agreement with that of rat BVR (Tyr97 mutant) ${ }^{7}$. Notably, the turnover rates were dramatically decreased relative to that of the wild-type protein by introduction of mutations at $\operatorname{Arg} 185$ (R185A and R185K): $k_{\text {cat }} / K_{\mathrm{m}}$ was reduced to $\sim 0.25 \%$, whereas their affinity for the substrate $\left(K_{\mathrm{m}}\right)$ was not significantly altered. These results also indicated that the guanidine group of the arginine residue in this position was invariant for the enzymatic reaction, as it was not able to substitute for positively charged amino group of the lysine residue. This arginine residue is completely conserved among BVR homologues, including rat (Arg171) and human BVR (Arg172) (Supplementary Fig. 2).

Next, we examined the enzymatic activity of rat and human BVRs by replacing the arginine at this site with other residues. As a result, in both rat and human BVRs, the $k_{\text {cat }} / K_{\mathrm{m}}$ values were remarkably decreased. In human BVR, the R172E and R172K mutations completely abolished the enzymatic activity. In the cofactor-bound state, although the position of the guanidine group of this arginine residue was almost identical among Syn, human, and rat BVRs (Supplementary Fig. 5), the conformation of its side chains had little variation (for example, torsion angles of $C \beta$ and $C \gamma$ atoms). Taken together, these data indicate that this arginine residue is the common catalytic residue of BVRs.

The BV binding to Syn BVR in solution states. To obtain further insight into the substrate-binding stoichiometry in solution, we measured BV binding to Syn BVR by optical titration. The BV absorption spectrum was slightly altered upon BVR binding in the presence of $\mathrm{NADP}^{+}$. Difference spectra (that is, in Syn BVR present minus Syn BVR absent) exhibited a negative peak at $650 \mathrm{~nm}$ and positive peaks at 420 and $740 \mathrm{~nm}$ (Fig. 6a). BV titration revealed that all of the difference peaks blue-shifted by $\sim 10 \mathrm{~nm}$. Peak amplitudes increased with BV concentration up to a twofold excess of BVR. The titration curve at $740 \mathrm{~nm}$ clearly indicated that two BV molecules bound to Syn BVR (Fig. 6b). This BV titration result agreed closely with the crystal structure. We also performed BV titration using the Syn BVR R185A mutant protein. The resultant titration curve confirmed that two BV molecules bound to Syn BVR R185A (Fig. 6c). Thus, the arginine residue in this position is not necessary for the two BV stacked binding, but is essential for the catalytic function.

To determine whether two-substrate binding is common among mammalian BVRs, we performed a titration in human and rat BVRs (Supplementary Fig. 6). The titration curve indicated that two BV molecules bound in both human and rat BVRs. Therefore, the mode of substrate binding is common among all BVRs.

\section{Discussion}

In this study, we experimentally determined the substrate-binding configurations in BVR concomitant with that of $\mathrm{NADP}^{+}$. To the 


\begin{tabular}{|c|c|c|c|}
\hline Variant & $k_{\text {cat }}\left(s^{-1}\right)$ & $K_{m}(\mu M)$ & $k_{\text {cat }} / K_{\mathrm{m}}\left(\mathrm{s}^{-1} \mu \mathrm{M}^{-1}\right)$ \\
\hline \multicolumn{4}{|c|}{ Synechocystis sp. PCC 6803} \\
\hline $\mathrm{Y} 102 \mathrm{~F}$ & $2.7 \pm 0.07$ & $4.0 \pm 0.3$ & 0.7 \\
\hline $\mathrm{T} 169 \mathrm{~A}$ & $13.1 \pm 0.5$ & $3.4 \pm 0.5$ & 3.9 \\
\hline S184A & $11.3 \pm 0.5$ & $1.9 \pm 0.4$ & 5.9 \\
\hline R185A & $0.7 \pm 0.02$ & $6.9 \pm 0.6$ & 0.1 \\
\hline K237A & $23.7 \pm 2.1$ & $4.8 \pm 1.3$ & 4.9 \\
\hline R246A & $11.0 \pm 0.6$ & $5.1 \pm 0.8$ & 2.2 \\
\hline \multicolumn{4}{|l|}{ Human } \\
\hline Wild-type & $94.8 \pm 31.4$ & $20.7 \pm 10.1$ & 4.6 \\
\hline Y98F & $16.8 \pm 0.5$ & $3.8 \pm 0.3$ & 4.4 \\
\hline \multicolumn{4}{|l|}{ Rat } \\
\hline Wild-type & $69.3 \pm 8.5$ & $4.6 \pm 1.1$ & 15.1 \\
\hline Y97F & $13.6 \pm 0.6$ & $11.7 \pm 1.0$ & 1.2 \\
\hline $\mathrm{R} 171 \mathrm{~A}$ & $6.0 \pm 0.2$ & $24.4 \pm 1.5$ & 0.2 \\
\hline R171E & $0.7 \pm 0.2$ & $45.7 \pm 15.4$ & 0.02 \\
\hline $\mathrm{R} 171 \mathrm{~K}$ & $14.7 \pm 8.5$ & $163.3 \pm 103.2$ & 0.1 \\
\hline
\end{tabular}

best of our knowledge, parallel stacking of two tetrapyrrole molecules is quite rare binding configuration. Many multitetrapyrrole proteins are present, including photosynthesis complexes $^{15,16}$ and cytochromes ${ }^{17}$. However, even if the tetrapyrrole compounds are located close to each other in these proteins, the two tetrapyrrole rings are not stacked, and the propionate side chain moieties are crossed or stacked in most cases. By contrast, in the BVR structure revealed in this study, the tetrapyrrole rings in the proximal and distal BVs are nearly parallel. Furthermore, the tetrapyrrole rings are located close to each other (within $\sim 4 \AA$ ).

Given that the enzymatic activity of BVRs is inhibited by excess $\mathrm{BV}^{18}$, this unprecedented configuration raises the possibility that this structure reflects a substrate-inhibited state. However, in the procedure of the co-crystallization in this study, we never added a molar excess of substrate, and the crystallization drops contained equimolar amounts of BVR and BV. Indeed, the enzymatic reaction of $\mathrm{BV}$ reduction could be induced to occur, even in the crystalline state, by addition of NADPH (see Fig. 4a). The colour of the crystal $10 \mathrm{~min}$ after the reaction was quite similar to that of a solution mixture of BV and BR (see Figs 4a,b), even though the crystal gradually melted. Moreover, we confirmed that BV could be rapidly (as in the mixing time) reduced to BR by addition of NADPH in a solution identical to the one used for crystallization. Thus, the structure presented here represents the active state.

The mutagenesis and kinetics experiments revealed that an arginine residue that is completely conserved among BVRs is probably the proton donor residue (see Table 2 and Supplementary Fig. 1). Intriguingly, Arg185 is located far from the $\mathrm{C} 10$ double bond of the reducing site of the proximal $\mathrm{BV}$ $(>5.7 \AA)$ and the distal BV $(>6.0 \AA$ ) (see Fig. $5 \mathrm{~b})$. That is, the proton is not able to transfer directly to the reducing site. Instead, the guanidine group of Arg185 formed hydrogen bonds with the carboxy group of the propionate side chain of the proximal $\mathrm{BV}$ (within $2.8 \AA$ in all molecules of the asymmetric unit); thus, the $\operatorname{Arg} 185$ must first donate its proton to the propionate side chain of the proximal BV.
Because of their chi-angle rotation restraints, it would be extremely difficult for the propionate side chains of the proximal $\mathrm{BV}$ to transfer the proton to reduced site. The parallel stacked configuration of the proximal and distal BVs indicates that the proton from Arg185 in the propionate side chain of the proximal BV can easily transfer to the propionate side chain in the distal $\mathrm{BV}$. Although the propionate side chain of the distal BV is also located close to $\operatorname{Arg} 185(\sim 3.1 \AA)$, and probably the proton could be directly transferred from Arg185, a hydrogen bond may not be formed due to the parallel configuration between the amino acid residue and the side chain. The propionate side chain in the distal $\mathrm{BV}$ is in a suitable location for transferring the proton to the proximal BV: it can approach within $2.6 \AA$ of the $\mathrm{C} 10$ carbon, as well as the pyrrole nitrogen in the B-ring in the proximal BV $(<2.5 \AA)$, via rotation of the propionate side chain of the distal BV.

Taken together, these observations suggest a plausible proton pathway for conversion of $\mathrm{BV}$ to $\mathrm{BR}$ (Fig. 7): a hydride from $\mathrm{NAD}(\mathrm{P}) \mathrm{H}$ is directly transferred to the C10 double bond of the proximal BV, initiating the reduction of the proximal BV. The propionate side chain of the proximal BV accepts a proton from Arg185, thereby abolishing the electrostatic interaction between Arg185 and the propionate and increasing the flexibility of the side chain. Subsequently, the proton is transferred to the propionate side chain in the distal $\mathrm{BV}$, and the protonated propionic acid group in the distal BV moves toward the reducing site in the proximal BV to transfer the proton. Although the exact site to be transferred to the proton could not be determined by the present structure, the B-ring in the proximal BV is the most reasonable site since the propionate side chain in the distal $B V$ can be located close to the B-ring in the proximal BV. Finally, the proximal BV accepts the proton to produce BR.

The reaction mechanism proposed here could explain not only why BVR strictly requires the propionate as tetrapyrrole side chain ${ }^{11}$, but also why a carboxyl group with a dissociable proton on the propionate side chain is essential ${ }^{12}$. Mantle's group performed sophisticated kinetic analysis of BVR, and found that 

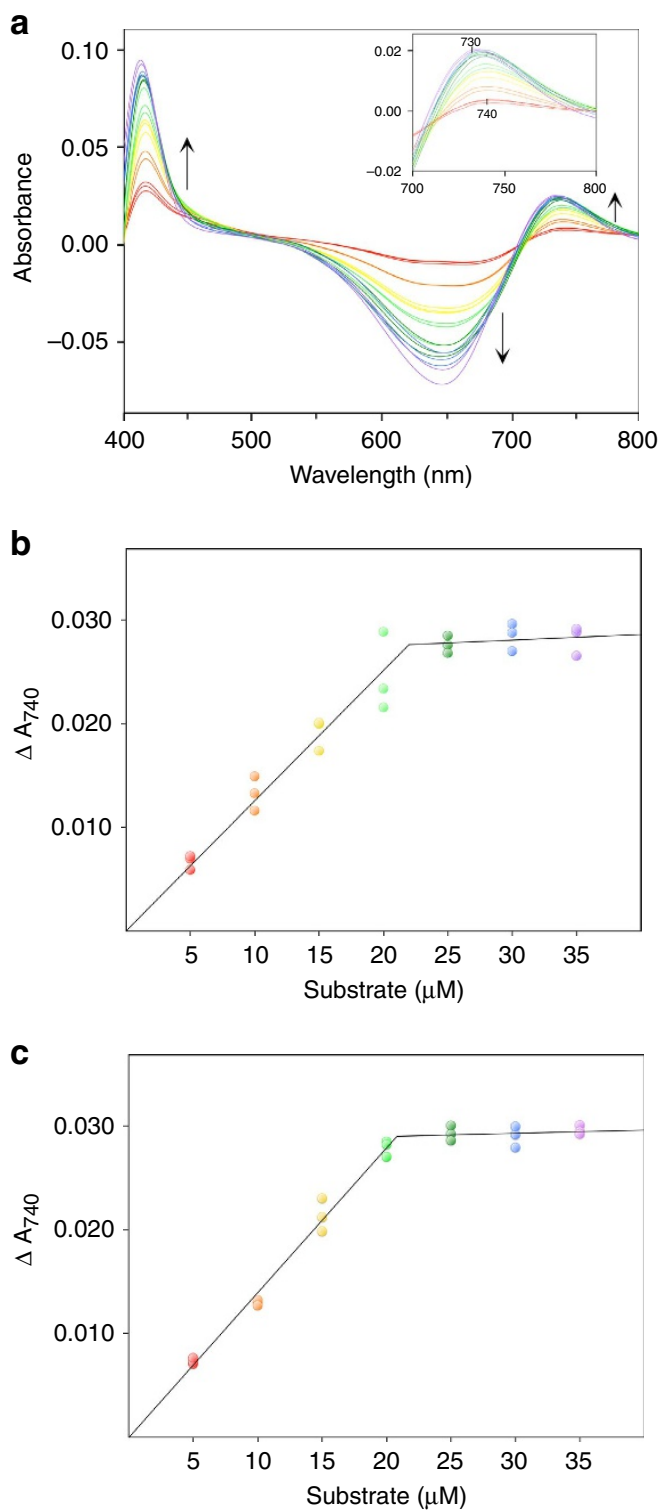

Figure 6 | Determination of the stoichiometry of BV binding to Syn BVR. (a) BV titration of Syn BVR $(10 \mu \mathrm{M})$, as determined from the difference absorption spectra acquired in the presence of $\mathrm{NADP}^{+}$. Arrows indicate trends of spectral changes. (b) Titration curve of Syn BVR observed at $740 \mathrm{~nm}$. (c) Titration curve of the Syn BVR R185A mutant. Measurements were performed in triplicate.

Syn BVR obeys an ordered steady-state kinetic mechanism, with $\mathrm{NADPH}$ the first to bind and $\mathrm{NADP}^{+}$the last to dissociate ${ }^{14}$. Based on this concept, we proposed a reaction mechanism for BVR. Following NADPH binding, the reaction is likely to start with hydride transfer from $\mathrm{NAD}(\mathrm{P}) \mathrm{H}$, because in time-course ultraviolet-visible spectra we have never observed a peak derived from $\mathrm{BVH}^{+}$, as seen in other BV-related enzymes ${ }^{19,20}$. The propionate side chain in another tetrapyrrole compound, protochlorophyllide, serves as proton donor for its self-reduction at the $\mathrm{C} 17=\mathrm{C} 18$ double bond to form chlorophyllide $a^{21}$. Thus, it is feasible that the proton donation function of the propionate side chain of $\mathrm{BV}$ is involved in the reduction reaction. QM/MM calculations for human BVR (BVR-A) and a related enzyme (BVR-B) have shown that the water molecule might be a proton donor $^{22,23}$, although the working hypothesis is that BVR binds one biliverdin molecule. We hope that by assuming binding of two biliverdin molecules, the QM/MM calculations can be used to reveal the detailed reaction mechanisms of BVR.

A structural and mechanistic understanding of the BVR reaction may enable the development of new inhibitors that target severe jaundice. In particular, the active pocket, where two BVs bind with the unique stacked geometry, is a promising target for design of drugs with minimal risk of deleterious side effects.

\section{Methods}

Purification and crystallization of Syn BVR. The plasmid pET15b-Syn bvr ${ }^{24}$ was used to overproduce N-terminal hexa-histidine tagged Syn BVR in the C41(DE3) strain (Lucigen) of $E$. coli. The cells were grown in Terrific-broth (TB) medium containing ampicillin $\left(50 \mu \mathrm{g} \mathrm{ml}^{-1}\right)$ at $28^{\circ} \mathrm{C}$, and overproduction was sustained overnight under shaking at $28^{\circ} \mathrm{C}$ after induction with $1 \mathrm{mM}$ isopropyl $\beta$-D-thiogalactopyranoside (IPTG). The N-terminal His-tagged Syn BVR was purified using nickel affinity gel (Nacalai Tesque) according to the manufacturer's protocol. The non-tagged Syn BVR was prepared by digestion of the $\mathrm{N}$-terminal His-tagged Syn BVR with 10 units of thrombin (GE Healthcare) per milligram of the protein in PBS buffer $\left(10 \mathrm{mM} \mathrm{Na}_{2} \mathrm{HPO}_{4}, 1.8 \mathrm{mM} \mathrm{KH}_{2} \mathrm{PO}_{4}, 2.7 \mathrm{mM} \mathrm{KCl}\right.$ and $140 \mathrm{mM} \mathrm{NaCl}$ ) at $20^{\circ} \mathrm{C}$ for $48 \mathrm{~h}$. Non-tagged Syn BVR was separated from remaining His-tagged Syn BVR by mixing it again with the nickel affinity gel. The flow-through fraction containing the non-tagged Syn BVR was concentrated with a VIVASPIN filter (GE Healthcare), and applied onto a HiPrep 16/60 Sephacryl S-200 HR gel filtration column (GE Healthcare) equilibrated in PBS buffer. The elution profile was monitored at absorption at $280 \mathrm{~nm}$, and its purity was confirmed by SDS-PAGE.

Purified non-tagged Syn BVR in PBS buffer was crystallized by the hangingdrop vapour diffusion method. In the initial crystallization screening, drops consisting of $1 \mu \mathrm{l}$ protein solution mixed with $1 \mu \mathrm{l}$ precipitant solution. Final optimized crystallization conditions were as follows: $12 \mathrm{mg} \mathrm{ml}^{-1}$ protein in $50 \mathrm{mM}$ Tris- $\mathrm{HCl}$ ( $\mathrm{pH} 7.8$ ) containing $150 \mathrm{mM} \mathrm{NaCl}$ and the reservoir solution was composed of $15 \%$ PEG 4,000, $50 \mathrm{mM}$ Tris- $\mathrm{HCl}$ ( $\mathrm{pH} 7.25$ ), $0.2 \mathrm{M}$ sodium acetate and $0.2 \mathrm{mM}$ Cymal-2 (Hampton Research).

Data collection and structure determination of apo-Syn BVR. The crystals were cryoprotected with a reservoir solution containing $12.5 \%$ glycerol for a few seconds, and then flash-cooled in a cryostream. The complete apo-Syn BVR data set was collected on an ADSC Quantum 4R detector with synchrotron radiation at a wavelength of $1.000 \AA$ at BL38B1 beamline of SPring-8 (Hyogo, Japan). Intensity data were processed and scaled using the HKL2000 software package $^{25}$.

Gold derivatives were obtained by soaking apo-Syn BVR crystals for $2 \mathrm{~h}$ in reservoir solution containing $10 \mathrm{mM}$ potassium dicyanoaurate (I). The Au-soaked crystal was cryoprotected with reservoir solution containing $10 \%$ glycerol and flash-frozen in liquid nitrogen. X-ray diffraction data were collected at BL32XU beamline of SPring- 8 and processed using HKL2000. Experimental phases were obtained from the Au-soaked crystal by the single isomorphous replacement method coupled with anomalous scattering (SIRAS) using AutoSol in PHENIX ${ }^{26}$. The model was built manually in $\mathrm{COOT}^{27}$, and refined in PHENIX. Secondary structures were assigned using PROMOTIF ${ }^{28}$, and the geometry of the final model was analysed using MolProbity ${ }^{29}$. Data collection and refinement statistics are summarized in Table 1.

Structure determination of NADP ${ }^{+}$-bound Syn BVR. Cofactor-bound crystals were prepared by soaking crystals for $20 \mathrm{~h}$ in a reservoir solution containing $10 \mathrm{mM}$ $\mathrm{NADP}^{+}$. The NADP ${ }^{+}$-soaked crystal was transferred to a cryo-protectant solution containing $10 \%$ glycerol and flash-frozen in liquid nitrogen. The data sets were collected at BL44XU beamline of SPring- 8 and processed with the XDS package $^{30}$.

Because the NADP ${ }^{+}$-soaked Syn BVR crystals were nearly isomorphous with the apo-form crystal, the structure of NADP ${ }^{+}$-bound Syn BVR was determined using the structure of apo-Syn BVR (PDB ID: 5B3T). Refinement was done using PHENIX $^{26}$ and the model was revised manually in COOT ${ }^{27}$. In this step, the $\mathrm{NADP}^{+}$was clearly visible in the electron density map. After one cycle of water picking, energy minimization refinement, and temperature-factor refinements, the model of the $\mathrm{NADP}^{+}$was fitted to the $F_{\mathrm{o}}-F_{\mathrm{c}}$ difference map in the active pocket. Finally, energy minimization and temperature-factor refinements in PHENIX were applied to the model including $\mathrm{NADP}^{+}$. Refinement statistics are summarized in Table 1.

Structure determination of Syn BVR in complex with BV and NADP ${ }^{+}$. Syn $\mathrm{BVR}$ in complex with $\mathrm{BV}$ and $\mathrm{NADP}^{+}$was crystallized using the sitting-drop vapour-diffusion method. Crystallization drops made using Mosquito (TTP Labtech) consisting of $0.1 \mu \mathrm{l}$ protein solution and $0.1 \mu \mathrm{l}$ reservoir solution were equilibrated against $50 \mu \mathrm{l}$ reservoir solution. Initial crystallization trials were done with commercially available sparse-matrix screening kits. The bluish-green small crystals grew at $20^{\circ} \mathrm{C}$. Crystallization conditions were as follows: $20 \mathrm{mg} \mathrm{m}^{-1}$ 

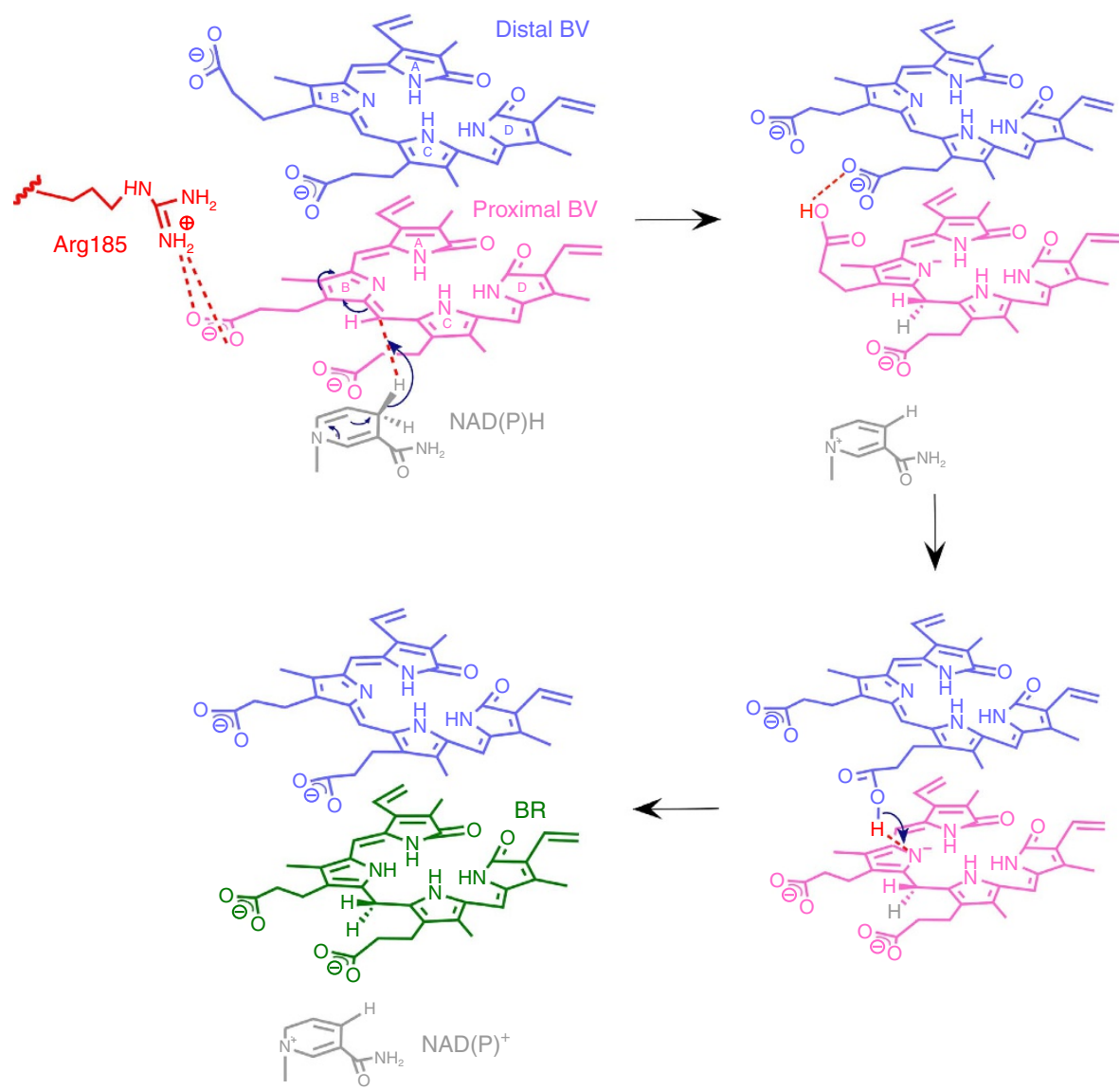

Figure 7 | Proposed mechanism of the BVR reaction. A hydride from NAD(P)H is directly transferred to the proximal BV, initiating the reduction of the proximal BV. The propionate side chain of the proximal BV accepts a proton from Arg185, subsequently, the proton is transferred to the propionate side chain in the distal BV, and the protonated propionic acid group in the distal BV moves toward the reducing site in the proximal BV to transfer the proton. Finally, the proximal BV accepts the proton to produce BR. Arrows in the nicotinamide and pyrrole rings indicate the electron flow, and in the other positions indicate the proton or hydride transfer.

$(0.52 \mathrm{mM})$ protein in PBS buffer containing $0.52 \mathrm{mM}$ BV; reservoir solution consisting of $19 \%$ PEG $4000,30 \mathrm{mM}$ Tris- $\mathrm{HCl}$ (pH 8.2), $50 \mathrm{mM} \mathrm{MgCl}_{2}$ and $10 \mathrm{mM}$ $\mathrm{BaCl}_{2}$. Crystals grew to maximum dimensions of $0.08 \times 0.05 \times 0.01 \mathrm{~mm}$.

The crystal was cryoprotected with the reservoir solution containing $20 \%$ glycerol and flash-frozen in liquid nitrogen. X-ray diffraction data were collected on a Rayonics MX300HE detector at BL44XU beamline of SPring-8 and processed with HKL2000. The crystal belongs to the monoclinic space group $P 2_{1}$, and a complete data set was collected to a resolution of $2.6 \AA$ (Table 1). The structure was solved by the molecular replacement method using the model of apo-Syn BVR (PDB ID: 5B3T) as the search probe, excluding solvent molecules. Rotational and translational searches with MOLREP ${ }^{31}$ in the CCP4 package $^{32}$ using the data in the resolution range 15.0-4.0 $\AA$ located four molecules in the asymmetric unit. The coordinates of the model and the temperature factors were refined using PHENIX ${ }^{26}$ and manually revised with $\mathrm{COOT}^{27}$. After several cycles of refinement and model adjustment, $\mathrm{BV}, \mathrm{NADP}^{+}$and water molecules were included in the model. The coordinates, topologies, and parameters of BV were generated using the PRODRG2 server $^{33}$. Diffraction data and refinement statistics are summarized in Table 1.

Expression and purification of rat BVR. The plasmid pET21a-rat $b v r^{34}$ was used to overproduce C-terminal His-tagged rat BVR in the BL21(DE3) strain (Novagen) of $E$. coli. The cells were grown overnight in $\mathrm{TB}$ medium containing ampicillin $\left(50 \mu \mathrm{g} \mathrm{ml}^{-1}\right)$ at $28^{\circ} \mathrm{C}$ without IPTG induction. The cells were collected by centrifugation, and the cell pellets were frozen at $-80^{\circ} \mathrm{C}$.

The C-terminal His-tagged rat BVR was purified using nickel affinity gel (Nacalai Tesque). After His-tagged rat BVR was eluted from the gel, the sample solution was exchanged to the PBS buffer by dialysis.

Expression and purification of human BVR. The human BVR gene was constructed from synthetic DNA (Eurofins Genomics) with codon optimization for E. coli. The human BVR gene was cloned into pET21a $(+)$ (Novagen) in E. coli strain BL21(DE3) (Novagen) to produce C-terminal His-tagged human BVR. His-tagged human BVR was expressed and purified as described for His-tagged rat BVR.
Site-directed mutagenesis. Site-directed mutagenesis was carried out using KOD-plus-Mutagenesis Kit (Toyobo), pET15b containing Syn BVR gene or $\operatorname{pET} 21 \mathrm{a}(+)$ containing human or rat BVR gene as a template, and the primers listed in Supplementary Table 1. Sequence analysis verified that the resultant constructs were free of errors.

Enzymatic assay. Syn BVR activity was measured as previously reported ${ }^{13}$ by monitoring the increase in absorbance at $450 \mathrm{~nm}$ (due to appearance of BR) using a V630BIO spectrometer (JASCO). Briefly, the reaction mixture contained $10 \mu \mathrm{g}$ purified BVR $(0.27 \mu \mathrm{M}), 100 \mathrm{mM}$ citrate buffer ( $\mathrm{pH} 5.75), 100 \mu \mathrm{M}$ NADPH and various concentration of $\mathrm{BV}$. The total volume was $1 \mathrm{ml}$, and the reaction was initiated by addition of NADPH. The Michaelis constant values for BV were determined using reaction mixtures containing various concentrations of $\mathrm{BV}$, and initial velocity data were analysed by non-linear curve fitting using GraphPad Prism 6 (GraphPad Software). The human and rat BVR assays used the same conditions as for Syn BVR, except that the buffer contained $100 \mathrm{mM}$ Tris- $\mathrm{HCl}$ ( $\mathrm{pH}$ 8.75).

Determination of protein and BV concentrations. Protein concentrations were determined by UV absorption using a molar extinction coefficient $\left(\varepsilon_{280}\right.$ values of $41,370 \mathrm{M}^{-1} \mathrm{~cm}^{-1}$ for the Syn BVR, $25,440 \mathrm{M}^{-1} \mathrm{~cm}^{-1}$ for the human BVR and $23,950 \mathrm{M}^{-1} \mathrm{~cm}^{-1}$ the for rat BVR, respectively) calculated from the amino acid sequence using the ExPASy ProtParam server ${ }^{35}$. BV was dissolved in a small amount of $0.1 \mathrm{~N} \mathrm{NaOH}$ and diluted into $100 \mathrm{mM}$ Tris- $\mathrm{HCl}(\mathrm{pH} \mathrm{7.8)}$; the concentration of $\mathrm{BV}$ was determined in $\mathrm{HCl}(5 \%) /$ methanol using an $\varepsilon_{696}$ value of $30,800 \mathrm{M}^{-1} \mathrm{~cm}^{-1}$ (ref. 13).

BV titration assay. BV binding stoichiometry was estimated by optical titrations in PBS buffer containing $5 \mathrm{mM} \mathrm{NADP}^{+}$at $28^{\circ} \mathrm{C}$. BV was added in $5 \mu \mathrm{M}$ increments to both the sample and the reference cuvettes; the former contained $10 \mu \mathrm{M}$ Syn BVR, whereas the latter contained no Syn BVR. Optical absorption spectra ranging from 400 to $800 \mathrm{~nm}$ were recorded at least 10 min after each 
addition of BV using a V630BIO spectrometer. Titration assays for human and rat BVR used the same conditions as for Syn BVR, except that the buffer contained $200 \mathrm{mM}$ Tris-HCl (pH 8.75).

Size-exclusion chromatography. Size-exclusion chromatography was conducted using Sephacryl 200 HR 16/60 (GE Healthcare) columns equilibrated with PBS buffer $\left(140 \mathrm{mM} \mathrm{NaCl}, 2.5 \mathrm{mM} \mathrm{KCl}, 10 \mathrm{mM} \mathrm{Na}_{2} \mathrm{HPO}_{4}, 1.8 \mathrm{mM} \mathrm{KH}_{2} \mathrm{PO}_{4}\right)$ at $20^{\circ} \mathrm{C}$. The calibration proteins [ $\beta$-amylase $(200 \mathrm{kDa})$, alcohol dehydrogenase $(150 \mathrm{kDa})$, BSA $(66 \mathrm{kDa})$, carbonic anhydrase $(29 \mathrm{kDa})$ and cytochrome $c(12.4 \mathrm{kDa})]$ were applied to the column.

Dynamic light scattering. Dynamic light scattering analysis was performed using a DynaPro99 Dynamic Light Scattering system (Protein Solutions). The wild-type Syn BVR $\left(1 \mathrm{mg} \mathrm{ml}^{-1}\right)$ was centrifuged at $20,400 \mathrm{~g}$ for $30 \mathrm{~min}$ to remove large aggregates prior to the experiments. Twenty readouts were averaged to obtain the mean and s.d. of sample molecular weights.

Enzymatic assay for NADH-dependent BVR activity. NADH-dependent BVR activity was measured by monitoring the increase in absorbance at $450 \mathrm{~nm}$. The reaction mixture contained $10 \mu \mathrm{g}$ purified BVR $(0.27 \mu \mathrm{M}), 100 \mathrm{mM}$ citrate buffer (pH 5.75), $700 \mu \mathrm{M}$ NADH, $20 \mu \mathrm{M} \mathrm{BV}$ and various concentration of sodium phosphate. Initial velocity data were analysed by non-linear curve fitting using GraphPad Prism 6 (GraphPad Software). In this measurement, great care was taken to control $\mathrm{pH}$, because the activity is highly sensitive to $\mathrm{pH}$.

Thermal-shift assays of Syn BVR. Thermal-shift assays were performed on a CFX96 Real-Time PCR Cycler (Bio-Rad Laboratories). In these experiments, $1 \mu \mathrm{l}$ of SYPRO Orange (Sigma-Aldrich, diluted from 5,000 $\times$ stock into Milli-Q), $8 \mu \mathrm{l}$ protein $\left(1.0 \mathrm{mg} \mathrm{ml}^{-1}\right)$, and $1 \mu \mathrm{l}$ buffer ( $1 \mathrm{M}$ citrate buffer ( $\mathrm{pH}$ 5.75)) were mixed on ice in a white 96-well PCR plate (Bio-Rad Laboratories). To evaluate the effect of NADP ${ }^{+}$binding, $1 \mu \mathrm{l}$ of 5,000 $\times$ SYPRO Orange, $8 \mu \mathrm{l}$ protein $\left(1.0 \mathrm{mg} \mathrm{ml}^{-1}\right)$ and $1 \mu \mathrm{l}$ buffer $\left(1 \mathrm{M}\right.$ citrate buffer $(\mathrm{pH} 5.8)$ ) containing $10 \mathrm{mM} \mathrm{NADP}^{+}$were mixed. Fluorescence was measured as temperature increased from 25 to $85^{\circ} \mathrm{C}$ in $0.5^{\circ} \mathrm{C}$ steps (excitation, $450-490 \mathrm{~nm}$; detection, $560-580 \mathrm{~nm}$ ). All measurements were performed three times. Data evaluation and melting-point determination were conducted using the Bio-Rad CFX Manager software.

Data availability. Coordinates and structure factors for apo-, $\mathrm{NADP}^{+}$-bound and $\mathrm{BV}-\mathrm{NADP}^{+}$complex forms of BVR have been deposited in the Protein Data Bank under accession codes 5B3T, 5B3U and 5B3V, respectively. All additional experimental data are available from the corresponding author on reasonable request. The PDB accession codes 1LC3 and 2H63, UniProt accession codes P53004, P46844, P72782, Q8YPP4 and Q9CY64 were used in this study.

\section{References}

1. Singleton, J. W. \& Laster, L. Biliverdin reductase of guinea pig liver. J. Biol. Chem. 240, 4780-4789 (1965).

2. Kuenzle, C. C., Weibel, M. H., Pelloni, R. R. \& Hemmerich, P. Structure and conformation of bilirubin. Opposing views that invoke tautomeric equilibria, hydrogen bonding and a betaine may be reconciled by a single resonance hybrid. Biochem. J. 133, 364-368 (1973).

3. McDonagh, A. F., Palma, L. A. \& Lightner, D. A. Blue light and bilirubin excretion. Science 208, 145-151 (1980).

4. Baranano, D. E., Rao, M., Ferris, C. D. \& Snyder, S. H. Biliverdin reductase: a major physiologic cytoprotectant. Proc. Natl Acad. Sci. USA 99, 16093-16098 (2002).

5. Sedlak, T. W. et al. Bilirubin and glutathione have complementary antioxidant and cytoprotective roles. Proc. Natl Acad. Sci. USA 106, 5171-5176 (2009).

6. Kikuchi, A. et al. Crystal structure of rat biliverdin reductase. Nat. Struct. Biol. 8, 221-225 (2001).

7. Whitby, F. G., Phillips, J. D., Hill, C. P., McCoubrey, W. \& Maines, M. D. Crystal structure of a biliverdin IX $\alpha$ reductase enzyme-cofactor complex. J. Mol. Biol. 319, 1199-1210 (2002).

8. Awruch, J., Tomaro, M. L., Frydman, R. B. \& Frydman, B. The specificity of biliverdin reductase. The reduction of biliverdin XIII isomers. Biochim. Biophys. Acta 787, 146-151 (1984).

9. Terry, M. J., Maines, M. D. \& Lagarias, J. C. Inactivation of phytochrome- and phycobiliprotein-chromophore precursors by rat liver biliverdin reductase. J. Biol. Chem. 268, 26099-26106 (1993).

10. Tomaro, M. L. et al. The specificity of biliverdin reductase. A study with different biliverdin types. Biochim. Biophys. Acta 791, 350-356 (1984).

11. Frydman, R. B. et al. Biliverdin reductase: substrate specificity and kinetics. Biochim. Biophys. Acta 916, 500-511 (1987).

12. Shang, L., Rockwell, N. C., Martin, S. S. \& Lagarias, J. C. Biliverdin amides reveal roles for propionate side chains in bilin reductase recognition and in holophytochrome assembly and photoconversion. Biochemistry 49, 6070-6082 (2010).

13. Schluchter, W. M. \& Glazer, A. N. Characterization of cyanobacterial biliverdin reductase. Conversion of biliverdin to bilirubin is important for normal phycobiliprotein biosynthesis. J. Biol. Chem. 272, 13562-13569 (1997).

14. Hayes, J. M. \& Mantle, T. J. The effect of $\mathrm{pH}$ on the initial rate kinetics of the dimeric biliverdin-IXalpha reductase from the cyanobacterium Synechocystis PCC6803. Febs J. 276, 4414-4425 (2009).

15. Nelson, N. \& Junge, W. Structure and energy transfer in photosystems of oxygenic photosynthesis. Annu. Rev. Biochem. 84, 659-683 (2015).

16. Shen, J. R. The structure of photosystem ii and the mechanism of water oxidation in photosynthesis. Annu. Rev. Plant Biol. 66, 23-48 (2015).

17. Mowat, C. G. \& Chapman, S. K. Multi-heme cytochromes--new structures, new chemistry. Dalton Trans. 21, 3381-3389 (2005).

18. Phillips, O. \& Mantle, T. J. Some kinetic and physical properties of biliverdin reductase. Biochem. Soc. Trans. 9, 275-278 (1981).

19. Tu, S. L., Gunn, A., Toney, M. D., Britt, R. D. \& Lagarias, J. C. Biliverdin reduction by cyanobacterial phycocyanobilin:ferredoxin oxidoreductase (PcyA) proceeds via linear tetrapyrrole radical intermediates. J. Am. Chem. Soc. 126, 8682-8693 (2004).

20. Unno, M. et al. Insights into the proton transfer mechanism of a bilin reductase pcyA following neutron crystallography. J. Am. Chem. Soc. 137, 5452-5460 (2015).

21. Muraki, N. et al. X-ray crystal structure of the light-independent protochlorophyllide reductase. Nature 465, 110-114 (2010).

22. Smith, L. J., Browne, S., Mulholland, A. J. \& Mantle, T. J. Computational and experimental studies on the catalytic mechanism of biliverdin-IXbeta reductase. Biochem. J. 411, 475-484 (2008).

23. Fu, G., Liu, H. \& Doerksen, R. J. Molecular modeling to provide insight into the substrate binding and catalytic mechanism of human biliverdin-IXalpha reductase. J. Phys. Chem. B 116, 9580-9594 (2012).

24. Watanabe, A. et al. Expression, purification and preliminary X-ray crystallographic analysis of cyanobacterial biliverdin reductase. Acta Crystallogr. Sect. F Struct. Biol. Cryst. Commun. 67, 313-317 (2011).

25. Otwinowski, Z. \& Minor, W. Processing of X-ray diffraction data collected in oscillation mode. Methods Enzymol. 276, 307-326 (1997).

26. Adams, P. D. et al. PHENIX: a comprehensive Python-based system for macromolecular structure solution. Acta Crystallogr. D Biol. Crystallogr. 66, 213-221 (2010).

27. Emsley, P., Lohkamp, B., Scott, W. G. \& Cowtan, K. Features and development of Coot. Acta Crystallogr. D Biol. Crystallogr. 66, 486-501 (2010).

28. Hutchinson, E. G. \& Thornton, J. M. PROMOTIF--a program to identify and analyze structural motifs in proteins. Protein Sci. 5, 212-220 (1996).

29. Chen, V. B. et al. MolProbity: all-atom structure validation for macromolecular crystallography. Acta Crystallogr. D Biol. Crystallogr. 66, 12-21 (2010).

30. Kabsch, W. Integration, scaling, space-group assignment and post-refinement. Acta Crystallogr. D Biol. Crystallogr. 66, 133-144 (2010).

31. Vagin, A. \& Teplyakov, A. An approach to multi-copy search in molecular replacement. Acta Crystallogr. D Biol. Crystallogr. 56, 1622-1624 (2000).

32. Collaborative Computational Project, No. 4. The CCP4 suite: programs for protein crystallography. Acta Crystallogr. D Biol. Crystallogr. 50, 760-763 (1994).

33. Schuttelkopf, A. W. \& van Aalten, D. M. PRODRG: a tool for high-throughput crystallography of protein-ligand complexes. Acta Crystallogr. D Biol. Crystallogr. 60, 1355-1363 (2004).

34. Sun, D. et al. Crystallization and preliminary X-ray diffraction analysis of a rat biliverdin reductase. Acta Crystallogr. D Biol. Crystallogr. 56, 1180-1182 (2000).

35. Gasteiger, E. et al. Protein Identification and Analysis Tools on the ExPASy Server 571-607 (Humana Press, 2005).

\section{Acknowledgements}

This work was supported by Platform for Drug Discovery, Informatics and Structural Life Science funded by the Ministry of Education, Culture, Sports, Science and Technology, Japan (MEXT). The synchrotron radiation experiments were performed on BL44XU, BL38B1 and BL32XU at SPring-8 with the approval of the Japan Synchrotron Radiation Research Institute (Proposal Nos. 2009A1183, 2009B1219, 2013AB6863, 2014AB6963, 2015AB6557, 2016AB6622 and 2016AB6655). This work was supported by the Programme to Disseminate Tenure Tracking (to K.W.) from Japan Science and Technology Agency (J.S.T.); by Grants-in-Aid of Scientific Research 16H04754 (to K.W.), 16 K07280 (to M.S.), and 23370052 (to K.F.) from MEXT; by Grants-in-Aid for Young Scientists 20770092 and 25840026 (to M.S.) from MEXT. We thank Dr E. Yamashita, Dr A. Higashiura, Dr K. Yamashita and Dr K. Hirata for their assistance with data collection at the SPring-8 synchrotron radiation facility (Hyogo, Japan). Expression plasmid of rat BVR kindly was gifted from Dr S. Hayashi of Nagasaki International University, Prof Y. Higashimoto of Kurume University School of Medicine and Prof M. Noguchi of Teikyo University. We thank N. Kaseda, N. Kamimura and Y. Motoyama of the University of Miyazaki for technical assistance. 


\section{Author contributions}

K.F., M.S. and K.W. conceived the project. M.S. and K.W. designed and conducted the experiments. H.T., K.H., H.K., T.N., Y.H., J.H., H.S., Y.T., M.S. and K.W. performed protein expression, purification and crystallization, and determined the crystal structure. H.T., Y.N., H.K., H.S., Y.T. M.S. and K.W. carried out the enzymatic analyses. H.T., T.Y., Y.S., M.S., Y.A., K.Y., K.F., M.S. and K.W. wrote the manuscript. All authors approved the final submitted manuscript.

\section{Additional information}

Supplementary Information accompanies this paper at http://www.nature.com/ naturecommunications

Competing financial interests: The authors declare no competing financial interests.

Reprints and permission information is available online at http://npg.nature.com/ reprintsandpermissions/
How to cite this article: Takao, H. et al. A substrate-bound structure of cyanobacterial biliverdin reductase identifies stacked substrates as critical for activity. Nat. Commun. 8, 14397 doi: 10.1038/ncomms14397 (2017).

Publisher's note: Springer Nature remains neutral with regard to jurisdictional claims in published maps and institutional affiliations.

(c) (i) This work is licensed under a Creative Commons Attribution 4.0 International License. The images or other third party material in this article are included in the article's Creative Commons license, unless indicated otherwise in the credit line; if the material is not included under the Creative Commons license, users will need to obtain permission from the license holder to reproduce the material To view a copy of this license, visit http://creativecommons.org/licenses/by/4.0/

(C) The Author(s) 2017 